\title{
Electrospray lonisation Mass Spectrometry (ESI-MS): a powerful tool for the evaluation of chiral recognition in host-guest complexation
}

\author{
H. Nierengarten ${ }^{1}$, E. Leize ${ }^{* 1}$, C. Garcia ${ }^{2}$, G. Jeminet ${ }^{2}$ and A. Van Dorsselaer ${ }^{1}$ \\ ${ }^{1}$ Laboratoire de Spectrométrie de Masse Bio-Organique, Université Louis Pasteur, CNRS UMR 7509, 1, rue Blaise Pascal, \\ 67008 Strasbourg Cedex, France \\ ${ }^{2}$ Synthèse, Électrosynthèse et Étude de Systèmes à Intérêt Biologique (S.E.E.S.I.B.), Université Blaise Pascal Clermont-Ferrand, \\ CNRS UMR 6504, 24, avenue des Landais, 63177 Aubière Cedex, France \\ * Corresponding author: leize@ chimie.u-strasbg.fr
}

\begin{abstract}
The chiral recognition properties of a series of synthetic crown ethers or cryptands (1-10) incorporating a chiral spiroacetal moiety in the polyether chain toward ammonium enantiomeric pairs (G1 or G2) have been evaluated by Electrospray Ionisation Mass Spectrometry (ESIMS). Isotopic-labelling has been used to distinguish one diastereomeric host-guest complex ion from the other. The reliability of this method has also been tested by studying the stability of the labelling and the influence of the labelling on the degree of chiral recognition. Various degrees of chiral recognition have emerged from this study.
\end{abstract}

\section{Introduction}

Since the pioneering synthesis of macrocyclic receptors by Pedersen, Lehn and Cram [1], host-guest associations have been intensively studied, especially in the field of chiral recognition [2]. Indeed, crown ethers and their derivatives represent one of the simplest model systems of the features occurring in biological processes. The principal aim of these chiral recognition studies is therefore a better understanding of the discrimination mechanisms so as to be able to develop new ways of asymmetric synthesis, another major application is the enantiomeric separation by liquid chromatography [2].

The general method of chiral recognition involves a stoichiomeric amount of a given chiral host and two enantiomeric guests $\mathrm{R}$ and $\mathrm{S}$. The degree of chiral recognition is then simply characterised by the ratio of the quantities, noted $(\mathrm{R}) /(\mathrm{S})$, of the two diastereomeric complexes which are formed with the given host-guest system at the thermodynamic equilibrium. If this ratio, $(\mathrm{R}) /(\mathrm{S})$ approaches 1 , the selectivity is weak, whereas if the $(\mathrm{R}) /(\mathrm{S})$ value is far from 1 , the degree and the direction of chiral recognition is significant. These chiral recognition properties in host-guest complexes are usually evaluated by analytical methods such as UV-vis, calorimetry or NMR [3]. Unfortunately, in this chemistry field, the amount of sample available is often very low, and the sensitivity of these classical analytical techniques is not well adapted. Therefore, we propose here the use of electrospray ionisation mass spectrometry (ESI-MS) [4] as an alternative.

\section{Experimental}

Positive ESI mass spectra were obtained on a ES triple quadrupole mass spectrometer Quattro II (Micromass, Altrincham, UK). The ES source was heated to $60^{\circ} \mathrm{C}$. The sampling cone voltage $(\mathrm{Vc})$ was set at 20 Volts to avoid any fragmentation processes. Sample solutions were introduced into the mass spectrometer source with a syringe pump (Harvard type 55 1111: Harvard Apparatus Inc., South Natick, MA, USA) with a flow rate of $5 \mu 1 . \mathrm{min}^{-1}$. Calibration was performed using protonated horse myoglobin. Scanning was performed from m/z 100 to 1000 in $10 \mathrm{~s}$, and several scans were summed to obtain the final spectrum.

Hosts 1-10 were synthesised and purified as described previously by Garcia et al. [5]. Guests G1 and G2 that frequently appear in the literature were prepared and labelled by conventional methods. Samples for ESI-MS were prepared by mixing the corresponding host and a 1:1 ratio of the enantiomeric pair G1 or G2 in methanol at $10^{-4} \mathrm{M}$. After stirring at room temperature for $1 \mathrm{~min}$, the solution was directly analysed by ESI-MS. For each 1:1 mixed guest solution, the value of the $(\mathrm{R}) /(\mathrm{S})$-enantiomer guests was measured in order to correct the observed $(\mathrm{R}) /(\mathrm{S})$ values determined for the chiral recognition experiments.

\section{Results and discussion}

In this paper, we describe the evaluation by ESI-MS of the chiral recognition properties of synthetic crown ethers and synthetic cryptands incorporating a chiral spiroacetal moiety 
Table I. (R)/(S) ratios obtained by ESI-MS for the diastereomeric complexes formed between hosts 1-10 and ammonium enantiomeric pairs G1 or G2, $(R)$ is unlabelled and (S) is labelled with deuterium.

(R)/(S) with G2
$(R) /(S)$ with G1

* $(\mathrm{R}) /(\mathrm{S})$ ratios obtained for the cross chiral experiments. In this case, $(\mathrm{R})$ is labelled with deuterium instead of $(\mathrm{S})$.

in the polyether chain (1-10) toward ammonium enantiomeric guests G1 and G2 (Tab. I and Fig. 1). In order to evaluate this ESI-MS approach, three aspects were investigated in our study: the possibility to quantify the different species present in thermodynamic equilibrium in solution, the detection of chiral diastereomeric ions in spite of the absence of mass differences using isotopic-labelling, and the reliability of our method.

ESI-MS has been successfully used for several characterisations of natural or synthetically non-covalent assemblies such as proteins [6] or supramolecular complexes [7]. Hostguest interactions in particular have been widely studied by ESI-MS [8]. Indeed, under carefully controlled experimental conditions, in particular, if the energy communicated to the gas-phase ions is controlled, ESI-MS has the ability to convey intact non-covalent ions directly from solution to gas phase without fragmentation. Ions observed in the mass spectrum therefore reflect the intact species present in thermodynamic equilibrium in solution.

Recently, much attention has been paid to the reliability of an ESI mass spectrum for the relative quantification of the different molecular species present in thermodynamic equilibrium in solution. We have already shown in the case of alkali metal-18C6 crown ether and alkali metal-[2.2.2] cryptate complexes that ESI-MS can yield the proportions

\section{- Phenylglycine methyl ester: G1}<smiles>COC(=O)[C@H](N[Cl+3])c1ccccc1</smiles>

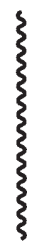<smiles>[CH2-]N[C@@H](C(=O)O[Na])c1ccccc1</smiles>

$\mathrm{R}$ $S$

\section{- Phenylglycinol: G2}<smiles>OCC(N[Cl+3])c1ccccc1</smiles>

R

$$
\{
$$<smiles>O=C(O)C(N[Cl+3])c1ccccc1</smiles>

S
Figure 1. Structures of the ammonium enantiomeric pairs G1 and G2. 
of the species present in solution, assuming that several conditions are respected [9]. The relative quantification by ESIMS is allowed when the species studied have similar structures, chemical functions, and close molecular weight in order to have similar desolvation energies and therefore close response factors. In our case, since the later conditions are respected for the diastereomeric host-guest complexes, proportions, and therefore chiral properties, can be quantified directly by measuring the ESI-MS relative intensities of the peaks corresponding to the (R) host-guest complex and the (S) host-guest complex.

To distinguish one diastereomeric host-guest complex ion from the other, one of the enantiomeric guests is labelled with deuterium. This approach has already been described by Sawada and co-workers [10]. In our case, each (S)-enantiomer was isotopically labelled with deuterium. The (S) phenylglycine methyl ester was labelled with three deuterium atoms and the (S)-phenylglycinol guest was labelled with two deuterium atoms (Fig. 1). Therefore, the two diastereomeric complex ions from a given host-guest combination differ by three or two mass units respectively in the mass spectrum. Hence, the proportions of the species can be quantified using the ESI mass spectrum. Moreover, diastereomer ions can also be distinguished by isotopiclabelling. Thus ESI-MS can be used successfully to evaluate the chiral recognition properties of our synthetic hosts.

The solutions were prepared by mixing in a stoichiometric ratio the (R)-unlabelled and (S)-labelled guest enantiomers, with a target crown ether host at $10^{-4} \mathrm{M}$ in methanol. After stirring at room temperature for one minute, the solution was directly analysed by ESI-MS (Fig. 2). Ions were obtained by the loss of the counter-ions $\mathrm{Cl}^{-}$and only monocharged peaks were observed. Therefore, only peaks with similar response factors were compared. The $(\mathrm{R}) /(\mathrm{S})$ ratios reported in table I are deduced from the relative intensities of the both diastereomeric complex ions (R)-unlabelled and (S)-labelled respectively, i.e. the complexes formed between the chiral crown ethers or the chiral cryptands (110) and ammonium pairs G1 or G2. The guests studied were chosen for their ability to complex crown and cryptand
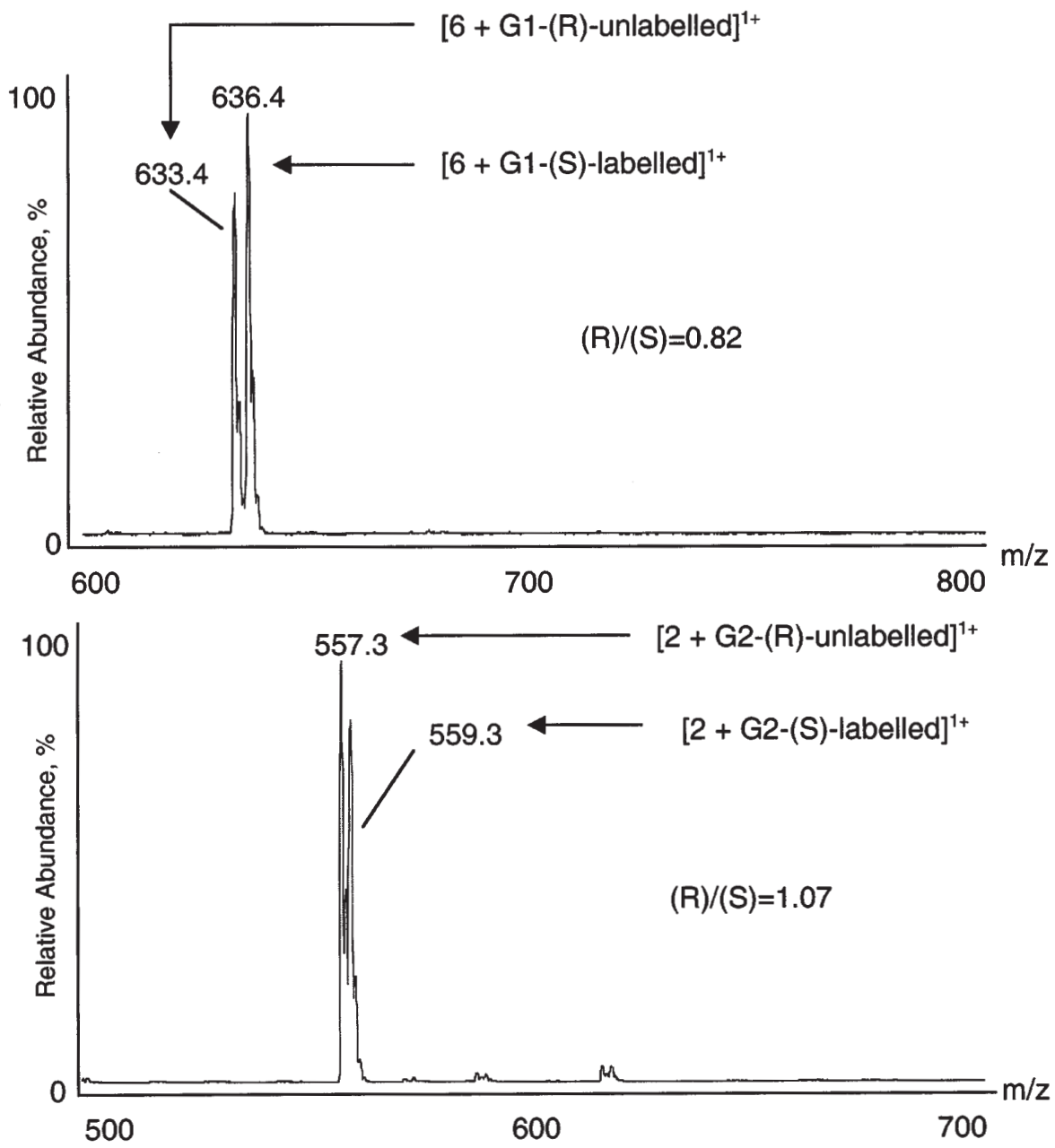

Figure 2. ESI mass spectra obtained with a 1/1/1 mixture of $6, \mathrm{G} 1-(\mathrm{R})$-unlabelled and G1-(S)-labelled and with a 1/1/1 mixture of 2, G2-(R)-unlabelled and G2-(S)-labelled. 

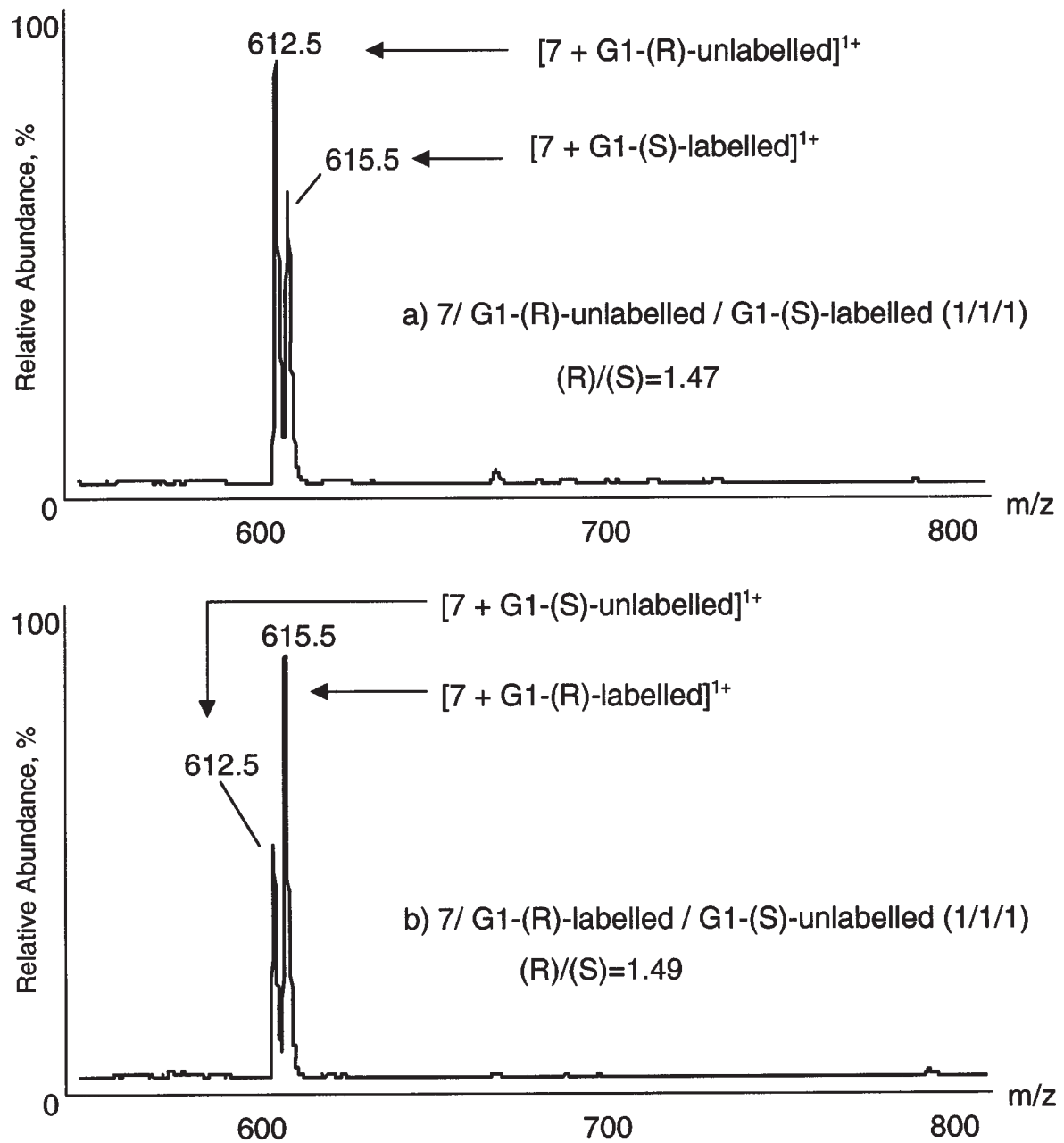

Figure 3. ESI mass spectra obtained for the cross-chiral correlation between 7 and G1.

derivatives owing to the presence of the ammonium function. In order to control the efficiency of our experiments, reference achiral hosts $18 \mathrm{C} 6$ and [2.2.2] cryptand have been tested under the same conditions with G1 and G2. The $(\mathrm{R}) /(\mathrm{S})$ values were equal to 1.00 , as expected for achiral hosts, indicating the reliability of the ESI-MS method.

Further, to check the stability of the labelling and its influence on the degree of chiral recognition, cross-chiral experiments have been performed. Here, the (R)-enantiomer guests have been isotopically labelled with deuterium. The ESI mass spectrum of the $1 / 1 / 1$ mixture of 7, G1-(R)labelled, and G1-(S)-unlabelled, obtained under the same conditions as the ES mass spectrum of the 1/1/1 mixture of 7, G1-(R)-unlabelled, and G1-(S)-labelled, showed that the same $(\mathrm{R}) /(\mathrm{S})$ ratios (1.47 and 1.49 respectively) were obtained for both solutions (Fig. 3). These findings indicate that the isotopic-labelling is stable (no H/D exchange) and has no effect on the degree of chiral recognition. The ESIMS results are therefore reproducible.
Our results have shown that several optically active macrocycles have chiral recognition properties (Tab. I). Indeed, as expected for the $C_{2}$ symmetric complexes [11], high $(\mathrm{R}) /(\mathrm{S})$ ratios have been recorded for the $C_{2}$ symmetric spiro-crown 4 with (R)-phenyl glycinol (1.41) and for the $C_{2}$ symmetric spiro-cryptand 7 with (R)-phenylglycine methyl ester (1.47). Another interesting observation was obtained for the $\mathrm{N}$-functionalisation, which created asymmetric structures (2, 3, 5, 6 crown ethers and 8, 9, 10 cryptands). This functionalisation did not markedly change the complexation for bicyclic systems $((\mathrm{R}) /(\mathrm{S})=1.0 ; 1.04 ; 1.05)$ but monocyclic polyethers were highly sensitive to this $\mathrm{N}$-branched function $((\mathrm{R}) /(\mathrm{S})=1.07 ; 1.23 ; 1.24 ; 1.15)$.

\section{Conclusion}

ESI-MS has been found to be a very informative and powerful tool for the study of host-guest chiral recognition, 
assuming that several experimental parameters are controlled. The sensitivity, the high speed and the small sample requirements suggest that ESI-MS may become a very attractive method for the study of chiral discrimination.

\section{Acknowledgements}

AVD, EL and HN acknowledge the TMR-Project EU Nr ERBFMRXCT980226 on Nanometer Size Metallic Complexes for the financial support of the ESMS work.

\section{References}

1. Pedersen, C. J. J. Am. Chem. Soc. 1967, 89, 7017; Lehn, J.-M. Struct. Bonding 1973, 161, 1; Cram, D. J. Science 1988, $240,760$.

2. Zhang, X. X.; Bradshaw, J. S.; Izatt, R. M. Chem. Rev. 1997, 97, 3313-3361.

3. Wang, T.; Bradshaw, J. S.; Izatt, R. M. J. Heterocyclic Chem. 1994, 31, 1097-1114.

4. Fenn, J. B.; Mann, M.; Meng, C. K.; Wong, S. F.; Whitehouse, C. M. Science 1989, 246, 64-71.

5. Garcia, C.; Pointud, Y.; Jeminet, G.; Dugat, D.; Beltran, J.-L. Tetrahedron: Asymmetry 1998, 9, 4253-4265; Garcia, C.;
Pointud, Y.; Jeminet, G.; Dugat, D. Tetrahedron Lett. 1999, 40, 4993-4996.

6. Loo, J. A. Mass Spectrom. Rev. 1997, 16, 1-23; Potier, N.; Barth, P.; Tritsch, D.; Biellmann, J.-F.; Van Dorsselaer, A. Eur. J. Biochem, 1997, 243, 274-282; Rogniaux, H.; Van Dorsselaer, A.; Barth, P.; Biellmann, J.-F.; Barbanton, J.; van Zandt, M.; Chevrier, B.; Howard, E.; Mitschler, A.; Potier, N.; Urzhumtseva, L.; Moras D.; Podjarny, A. J. Am. Soc. Mass Spectrom. 1999, 10, 635-647.

7. Leize, E.; Van Dorsselaer, A.; Krämer, R.; Lehn, J.-M. J. Chem. Soc., Chem. Commun. 1993, 990-993; Russel, K. C.; Leize, E.; Van Dorsselaer, A.; Lehn, J.-M. Angew. Chem. Int. Ed. Engl. 1994, 34, 209; Hopgartner, G.; Piguet, C.; Henion, J. D.; Williams, A. F. Helv. Chim. Acta 1993, 76, 1759-1766.

8. Blair, S. M.; Kempen, E. C.; Brodbelt, J. S. J. Am. Mass Spectrom. 1998, 9, 1049-1059. Vincenti, M. J. Mass Spectrom. 1995, 30, 925-939.

9. Leize, E.; Jaffrezic, A.; Van Dorsselaer, A. J. Mass Spectrom. 1996, 31, 537-544.

10. Sawada, M.; Takai, Y.; Keneda, T.; Arakawa, R.; Okamoto, M.; Doe, H.; Matsuo, T.; Naemura, K.; Hirose, K.; Tobe, Y. J. Chem. Soc., Chem. Comm. 1996, 1735-1736.

11. Garcia, C.; Guyot, J.; Jeminet, G.; Leize, E.; Nierengarten, H.; Van Dorsselaer, A. Tetrahedron Lett. 1999, 40, 4997-5000. 